\title{
ENERGY DECAY OF A LINEAR HYPERBOLIC EQUATION WITH LOCALLY DISTRIBUTED DAMPING
}

\author{
E. Cabanillas Lapa - J. Bernui - Z. Huaringa
}

ABSTRACT.- Decay estimates for the energy are derived for a linear hyperbolic equation with time-dependent coefficients.

\section{INTRODUCTION}

Let $\Omega$ a bounded domain in $\Re^{N}(N \geq 1)$ having a smooth boundary $\Gamma=\partial \Omega$. We fix $x_{0} \in R^{N}$. Let $a(x, t)$ be a nonnegative bounded function.

Let us consider the following linear hyperbolic equation.

$$
\mid \begin{array}{ll}
K(x, t) u^{\prime \prime}-\Delta u+\mathrm{a}(x, t) u^{\prime}=0 & \text { in } Q=\Omega \times] 0, T[ \\
u=0 & \text { on } \Sigma=\Gamma \times] 0, T[ \\
u(0)=u_{0}, u^{\prime}(0)=u_{1} & \text { on } \Omega
\end{array}
$$

Under the assumptions that $K(x, t) \geq h_{0}>0, \forall(x, t) \in Q$ and

$$
a(x, t)-\left|\frac{\partial K}{\partial t}(x, t)\right| \geq \varepsilon_{0}>0, \forall \times \in \omega
$$

Where $\omega$ is a neighborhood of $\overline{\Gamma_{0}}$,

$$
\Gamma_{0}=\{x \in \Gamma: \mathrm{m}(x) \cdot v(x) \geq 0\}
$$

being $v(x)$ the unit outward normal at $x \in \Gamma$ and $\mathrm{m}(x)=x-x_{0}$, we prove the exponential decay of weak solutions to problem (1.1).

The case $K(x, t)=1$ has been studied by many authors: M. Nakao [6]. L.R. Tcheougoné Tebou [7], P. Martínez [5]. Motived by the above results the authors of the present work investigated the case with time dependent coefficients. 


\section{PRELIMINARIES}

The function spaces we use are all standard and the definition of them is unified. We shall denote the following

$$
\begin{gathered}
(u, v)=\int_{\Omega} u(x) v(x) d x,|u|^{2}=\int_{\Omega}|u(x)|^{2} d x \\
u^{\prime}=\frac{\partial u}{\partial t} \text { and } R=\max _{x \in \bar{\Omega}}\left\|x-x_{0}\right\|
\end{gathered}
$$

Let us state the general hypothesis

(A.1) $K \in W^{1, \infty}\left(0, \infty ; C^{1}(\bar{\Omega})\right), K^{\prime} \in L^{\prime}\left(0, \infty, L^{\infty}(\Omega)\right)$

$$
K(x, t) \geq h_{0}>0, \forall(x, t) \in Q
$$

$$
\begin{array}{ll}
\text { (A.2) } & a \in W^{1, \infty}\left(0, \infty ; C^{-1}(\bar{\Omega})\right) \cap W^{2, \infty}\left(0, \infty ; L^{\infty}(\Omega)\right) \\
& a \in L^{1}\left(0, \infty ; L^{\infty}(\Omega)\right), a(x, t) \geq a_{0}>0 \text { in } Q \\
\text { (A.3) } \quad a(x, t)-\left|K^{\prime}(x, t)\right| \geq \varepsilon_{0}>0, \forall \times \in \omega
\end{array}
$$

We use the following well known lemma without the proof in this paper.

Lemma 2.1.- (Haraux [2]) Let $E:[0, \infty[\rightarrow[0, \infty$ [ be a nonincreasing locally absolutely continous funtion such that there are nonnegative constants $\beta$ and $A$ with

$$
\int_{s}^{\infty} E(t)^{\beta+1} d t \leq A \quad E(S), \forall S \geq 0
$$

Then we have

$$
E(t) \leq\left\{\begin{array}{l}
E(0) e^{1-\frac{t}{A}}, \forall t \geq 0, \text { if } \beta=0 \\
{\left[A\left(1+\frac{1}{\beta}\right)\right]^{1 / \beta} t^{1 / \beta}, \text { if } \beta>0}
\end{array}\right.
$$

\section{STATEMENT OF THE RESULTS}

In this section, we state main theorems.

\section{Theorem 3.1}

Let $u_{0} \in H_{0}^{1}(\Omega) \cap H^{2}(\Omega)$ and $u_{1} \in H_{0}^{1}(\Omega)$. Under assumptions $(A .1)-(A .3)$, for each $T>0$, the problem (1.1) admits a unique strong solution 


$$
\begin{gathered}
u: \Omega \times] 0, T[\rightarrow R \text { such that } \\
u_{0} \in L^{\infty}\left(0, T ; H_{0}^{1} \cap H^{2}\right), u^{\prime} \in L^{\infty}\left(0, T ; H_{0}^{1}\right), u^{\prime \prime} \in L^{\infty}\left(0, T ; L^{2}(\Omega)\right)
\end{gathered}
$$

Now, we present a result on stability of strong solutions wich will be extended to weak solutions .

\section{Theorem 3.2}

Let us consider the energy

$$
E(t)=\frac{1}{2}|\sqrt{K} u(t)|^{2}+\frac{1}{2}|\nabla u(t)|^{2}
$$

Under hypothesis of theorem 3.1, with $\nabla K . \mathrm{m} \geq 0 \forall \times \in \Omega$ the energy (3.2) determined by the strong solutions $u$ decays exponentially, that is for some constants $c>0$ and $r>0$

$$
E(t) \leq C E(0) e^{-\gamma t}, \quad \forall t \geq 0
$$

\section{Proof}

First we remark that is sufficient to prove the estimate (3.3) where the initial conditions verify

$$
\left\{u_{0}, u_{1}\right\} \in\left(H_{0}^{1}(\Omega) \cap H^{2}(\Omega)\right) \times H_{0}^{1}(\Omega)
$$

Then an easy density argument give the result for all initial condition in $H_{0}^{1}(\Omega) \times L^{2}(\Omega)$

We verify that (1.1) is a dissipative problem.

\section{Theorem 3.3}

Suppose that $u_{0} \in H_{0}^{1}(\Omega), u_{1} \in L^{2}(\Omega)$ and that assumptions (A1), (A2) hold. Then (1.1) has a unique weak solution $\mathrm{u}: \Omega \times] 0, T[\rightarrow R$ in the space

$$
C\left(0, T ; H_{0}^{1}(\Omega)\right) \cap C^{1}\left(0, T ; L^{2}(\Omega)\right)
$$

Furthermore, theorem (3.2) holds for the weak solution $u$.

\section{Lemma 3.1.-}

$$
\forall 0 \leq S<T<\infty: E(S)-E(T)=-\int_{S}^{T} \int_{\Omega}\left(a-\frac{1}{2} K^{\prime}\right)\left(u^{\prime}\right)^{2} d x d s
$$




\section{Proof.-}

We multiply (1.1) by $u^{\prime}$ and we integrate by parts on $\Omega \times[S, T]$, observing first that

$$
\left(K u^{\prime \prime}, u^{\prime}\right)=\frac{1}{2} \frac{d}{d t}\left|\sqrt{K} u^{\prime}\right|^{2}-\frac{1}{2} \int_{\Omega} K^{\prime}\left(u^{\prime}\right)^{2} d x .
$$

We obtain

$$
\int_{S}^{T}\left(K u^{\prime \prime}-\Delta u, u^{\prime}\right) d s=\left.\left(\frac{1}{2}\left|\sqrt{K} u^{\prime}\right|^{2}+\frac{1}{2}|\nabla u|^{2}\right)\right|_{S} ^{T}+\int_{S}^{T} \int_{\Omega}\left(a-\frac{1}{2} K^{\prime}\right)\left(u^{\prime}\right)^{2} d x d s
$$

Therefore

$$
E(T)-E(S)=-\int_{S}^{T}\left(\left(a-\frac{1}{2} K^{\prime}\right),\left(u^{\prime}\right)^{2}\right) d s
$$

Remark.- We deduce from (3.4) and (A.3) that.

$$
\int_{S}^{T}\left(\left(a-K^{\prime}\right), u^{2}\right) d s \leq E(S)-E(T)
$$

Lemma 3.2.- Let $q \in\left[\mathrm{W}^{1, \infty}(\Omega)\right] \cap \alpha \in \mathbb{R}$ and $\zeta \in \mathrm{W}^{1, \infty}(\Omega)$. We have the identities

$$
\begin{aligned}
& \left.\left(k u^{\prime}, 2 q \cdot \nabla u+\alpha u\right)\right|_{S} ^{T}+\int_{S}^{T} \int_{\Omega}(\text { divq }-\alpha)\left\{\left|\sqrt{K} u^{\prime}\right|^{2}-|\nabla u|^{2}\right\} d x d t+ \\
& +\int_{S}^{T}\left(\left(a-K^{\prime}\right) u^{\prime}, 2 q \cdot \nabla u+\alpha u\right) d t+\int_{S}^{T}\left(\nabla K \cdot q, u^{\prime 2}\right) d t \\
& +2 \int_{S}^{T} \int_{\Omega} \frac{\partial q_{K}}{\partial x_{J}} \frac{\partial u}{\partial x_{J}} \frac{\partial u}{\partial x_{J}} d x d t=\int_{S}^{T} \int_{\Gamma} q \cdot v\left(\frac{\partial u}{\partial v}\right)^{2} d \Gamma d t \\
& \left(K u^{\prime}, u\right)_{S}^{T}-\int_{S}^{T} \int_{\Omega} \zeta\left\{\left|\sqrt{K} u^{\prime}\right|^{2}-|\nabla u|^{2}\right\} d x d t+ \\
& +\int_{S}^{T}(u \nabla u, \nabla \zeta) d t+\int_{S}^{T}\left(\left(a-K^{\prime}\right) u^{\prime}, \zeta u\right) d t=0 .
\end{aligned}
$$

The proof of Lemma 3.2 is based on standard multipliers technique. The interested reader should refer to Lions [4] or Komornik [3].

Throughout the remaining part of this work positive constants will be denoted by $\mathrm{C}$ and will change from line to line.

In order to prove (3.3) we proceed in two steps.

Step 1. Appliying (3.5) with $q(x)=m(x), \alpha=n-1$ observing that $\operatorname{div}(m)=N$ and using (3.2) we get 


$$
\begin{gathered}
\left.\left(K u^{\prime}, 2 m . \nabla u+(N-1) u\right)\right|_{S} ^{T}+\int_{S}^{T} \int_{\Omega}\left(\left|\sqrt{K} u^{\prime}\right|^{2}-|\nabla u|^{2}\right) d x d t \\
+\int_{S}^{T}\left(\left(a-K^{\prime}\right) u^{\prime}, 2 m . \nabla u+(N-1) u\right) d t+\int_{S}^{T} \int_{\Omega} \nabla K \cdot m\left(u^{\prime}\right)^{2} d x d t \\
+2 \int_{S}^{T} \int_{\Omega}|\nabla u|^{2} d x d t=\int_{S}^{T} \int_{\Gamma} m \cdot v\left(\frac{\partial u}{\partial v}\right)^{2} d \Gamma d t ; \\
2 \int_{S}^{T} E(t) d t=-\left(K u^{\prime}, 2 m . \nabla u+(N-1) u\right)_{S}^{T}- \\
\quad-\int_{S}^{T}\left(\left(a-K^{\prime}\right) u^{\prime}, 2 m . \nabla u+(N-1) u\right) d t \\
-\int_{S}^{T} \int_{\Omega}(\nabla K . m) u^{\prime 2} d x d t+\int_{S}^{T} \int_{\Gamma} m \cdot v\left(\frac{\partial u}{\partial v}\right)^{2} d \Gamma d t
\end{gathered}
$$

Since the energy is nonincreasing, using the result of komornik [3], we obtain

$$
\begin{aligned}
\left|-\left(K u^{\prime}, 2 m \cdot \nabla u+(N-1) u\right)\right|_{S}^{T} \mid \leq C E(s) & \\
\left|-\int_{S}^{T}\left(\left(a-K^{\prime}\right) u^{\prime}, 2 m \cdot \nabla u+(N-1) u\right) d t\right| & \leq C \int_{S}^{T}\left|E^{\prime}(t)\right|^{1 / 2}\left|E^{1 / 2}(t)\right| d t \\
& \leq c E(S)+\varepsilon \int_{S}^{T} E(t) d t, \quad \varepsilon>0
\end{aligned}
$$

If follows from (3.8) - (3.10) that

$$
\int_{S}^{T} E(t) d t \leq C E(S)+R \int_{S}^{T} \int_{\Gamma_{0}}\left(\frac{\partial u}{\partial v}\right)^{2} d \Gamma d t
$$

To estimate the last term in (3.11) we utilize (3.7) with $\zeta=\eta$ where $\eta \in \mathrm{W}^{1, \infty}(\Omega)$ is a function that

$$
\mid \begin{array}{cc}
0 \leq \eta \leq 1 \\
\eta=1 & \text { in } \hat{\omega} \\
\eta=0 & \text { in } \Omega \backslash \omega
\end{array} \quad ; \frac{|\nabla \eta|^{2}}{\eta} \in L^{\infty}(\omega)
$$

and $\hat{\omega}$ is open set in $\Omega$ with $\Gamma_{0} \subseteq \hat{\omega} \varsubsetneqq \omega$. First we have from (3.7) 


$$
\begin{aligned}
\int_{S}^{T} \int_{\Omega} \eta|\nabla u|^{2} d x d t & =\left.\left(K u^{\prime}, \eta u\right)\right|_{S} ^{T}-\int_{S}^{T}\left(\left(a-K^{\prime}\right) u^{\prime}, \eta u\right) d t \\
& +\int_{S}^{T} \int_{\Omega} \eta\left|\sqrt{K} u^{\prime}\right|^{2} d x d t-\int_{S}^{T}(u \nabla u, \nabla \eta) d t
\end{aligned}
$$

Simple calculations, using Young's ineguality show that

$$
\begin{aligned}
& \left|-\left(K u^{\prime}, \eta u\right)\right|_{S}^{T} \leq C E(S) \\
& \mid-\int_{S}^{T}\left(\left(a-K^{\prime}\right) \mathrm{u}^{\prime}, \eta u\right) d t \leq C E(S)+\varepsilon \int_{S}^{T} E(t) d t \quad, \quad \varepsilon>0 \\
& \left|-\int_{S}^{T}(u \nabla u, \nabla \eta) d t\right| \leq C \int_{S}^{T} \int_{\omega}|u|^{2} d t d x+\frac{1}{2} \int_{S}^{T} \int_{\Omega} \eta|\nabla u|^{2} \in d x d t \\
& \left.\left.\left|\int_{S}^{T} \eta \int_{\Omega} n\right| \sqrt{K} u^{\prime}\right|^{2} d x d t\left|\leq C \int_{S}^{T} \int_{\omega}\right| u^{\prime}\right|^{2} d x d t
\end{aligned}
$$

Reporting (3.13) - (3.16) in (3.12), we find

$$
\begin{aligned}
\frac{1}{2} \int_{S}^{T} \int_{\Omega} \eta\left|\nabla u^{\prime}\right|^{2} d t d x & \leq C E(S)+C \int_{S}^{T} \int_{w}\left(\left|u^{\prime}\right|^{2}+|u|^{2}\right) d x d t+ \\
& +\varepsilon \int_{S}^{T} E(t) d t, \quad \varepsilon>0
\end{aligned}
$$

Step 2.- We take a vector field $h \in\left[W^{1, \infty}(\Omega)\right]^{N}$ such that

$$
h=v \text { on } \Gamma_{0}, h . v \geq 0 \text { on } \Gamma \text { and } h=0 \text { on } \Omega \backslash \hat{w}
$$

Choosing $\alpha=0$ and $\mathrm{q}=\mathrm{h}$ in (3.6), we easily deduce

$$
\int_{S}^{T} \int_{T_{0}}\left(\frac{\partial u}{\partial v}\right)^{2} d \Gamma d t \leq C\left(E(S)+\int_{S}^{T} \int_{\hat{\omega}}\left|u^{\prime}\right|^{2}+|\nabla u|^{2}\right) d x d t+\varepsilon \int_{S}^{T} E(s) d t
$$

combining (3.18) and (3.17), we have $\forall \varepsilon>0$

$$
\int_{S}^{T} \int_{\Gamma_{0}}\left(\frac{\partial u}{\partial v}\right)^{2} d \Gamma d t \leq C\left(E(S)+c \int_{S}^{T} \int_{\omega}\left|u^{\prime}\right|^{2}+|\nabla u|^{2}\right) d x d t+\varepsilon \int_{S}^{T} E(t) d t \quad, \quad \forall \varepsilon>0
$$


We conclude that (3.19) and (3.1) that

$$
\int_{S}^{T} E(t) d t \leq C E(S)+C \int_{S}^{T} \int_{\hat{\omega}}\left(\left|u^{\prime}\right|^{2}+|\nabla u|^{2}\right) d x d t
$$

Now in under to absorb the last theorem of the right hand side of (3.20) we adapt a method introduced in Conrad and Rao [ 1 ]. To this end, we consider $z(t) \in H_{0}^{1}(\Omega)$ solution of

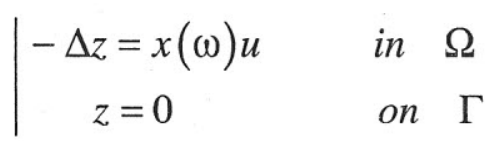

where $x(\omega)$ is the characteristic function of $\omega$.

It is easy to verify that $z^{\prime}$ is solution of the problem

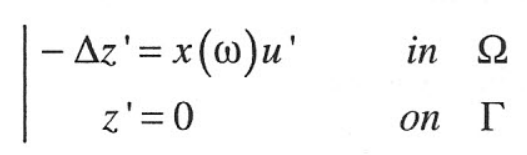

A simple computations gives

$$
\begin{aligned}
& |z| \leq C|u|_{L^{2}(u)} \\
& \left|z^{\prime}\right| \leq C\left|u^{\prime}\right|_{L^{2}(w)} \\
& (\nabla z, \nabla u)=|u|_{L^{2}(w)}
\end{aligned}
$$

Next, we multiply (1.1) by $z$, integrate by parts on $\Omega \times] S, T[$ and use (3.23), we obtain

$$
\int_{S}^{T} \int_{w}|u|^{2} d x d t=-\left.\left(K u^{\prime}, z\right)\right|_{S} ^{T}-\int_{S}^{T}\left(\left(a-K^{\prime}\right) u^{\prime}, z\right) d t+\int_{S}^{T}\left(K u^{\prime}, z^{\prime}\right) d t
$$

Now, we note that

$$
\begin{aligned}
& \left|\left(K u^{\prime}, z\right)\right| \leq C E(S) \\
& \left|\int_{S}^{T}\left(\left(a-K^{\prime}\right) u^{\prime}, z\right) d t\right| \leq C E(S)+\varepsilon \int_{S}^{T} E(t) d t, \quad \varepsilon>0 \\
& \left|\int_{S}^{T}\left(K u^{\prime}, z\right) d t\right| \leq C \int_{S}^{T} \int_{w}\left|u^{\prime}\right|^{2} d x d t+\varepsilon_{1} \int_{S}^{T} E(t) d t, \quad \varepsilon_{1}>0
\end{aligned}
$$


We deduce from the last three estimates and (3.24) that

$$
\int_{S}^{T} \int_{\omega}\left|u^{\prime}\right|^{2} d x d t \leq C E(S)+\varepsilon \int_{S}^{T} E(t) d t+C \int_{S}^{T} \int_{w}\left|u^{\prime}\right|^{2} d x d t
$$

Inserting (3.25) into (3.20) gives

$$
\int_{S}^{T} E(t) \leq C E(S)+C \int_{S}^{T} \int_{w}\left|u^{\prime}\right|^{2} d x d t
$$

Thanks to (A.3) we have

$$
\int_{S}^{T} \int_{\omega}\left|u^{\prime}\right|^{2} d x d t \leq \frac{1}{\varepsilon_{0}} \int_{S}^{T} \int_{\omega}\left(\mathrm{a}-\frac{1}{2} K^{\prime}\right) u^{\prime 2} d x d t \leq C E(S)
$$

Finally the application of lemma 2.1 implies (3.3). .

The proof of theorem 3.2 is completed.

Remark.- The proof of theorem (3.3) is achieved using a density argument. 


\section{BIBLIOGRAFIA}

[1] Conrad, F. \& Rao, B. Decay of Solution of Wave Equations in a star-shaped domain with non-linear boundary feedback. Asymptotic Analysis, 7, 159 - 177, (1993).

[2] Haraux A. Semi-groupes lineaires et équations d'évolution linéaires periodiques. Publications du Laboratoire d'Analyse Numérique. Université Pierre et Marie Curie, Paris. No 78011 (1978).

[3] Komornik, V. Exact Controllability and Stabilization. The Multiplier Method. RAM. Masson \& John Wiley. Paris. (1994).

[4] Lions, J. L. Contrólabilité exacte, perturbations et stabilisation des systemés distribués, N 1, RMA, Masson, Paris. (1988).

[5] Martínez, P. A New method to obtain decay rate estimates for dissipative systems with localized damping. Revista Mat. Compluterese Vol. 12, No 1, (1999).

[6] Nakao, M. Decay of solutions of the wave equations with a local degenerate dissipation. Israel J. Math; 95, 25-42 (1996).

[7] Tcheugoué Tébou L. R. On the decay estimates for the wave equation with a local degenerate or non degenerate dissipation, Portugal. Math. Vol. 55. Fasc. 3 - (1988). 